\section{Compromiso neuropático y autonómico en Enfermedad de Fabry: presentación de casos clínicos}

\author{
PAULA JARA ${ }^{1}$, JOSÉ MANUEL MATAMALA ${ }^{1,2,3,4, a}$, \\ RENATO VERDUGO ${ }^{1, b}$
}

\section{Neuropathy and Fabry's disease. Report of five cases}

Fabry's disease is an X-linked multisistemic lisosomal storage disorder caused by deficiency or absence in $\alpha$-Galatosidase A. Symptoms develop early in childhood with small fiber neuropathy, autonomic disorders and skin lesions (angiokeratomas). More severe in males, patients develop over years heart disease (hypertrophic cardiomyopathy, bradycardia), proteinuria, renal failure, transient ischemic attacks and stroke, associated with decreased life expectancy. We report five patients with Fabry's disease aged between 21 to 56 years and with family history. Neuropathic symptoms are described and neurophysiological testing findings of nerve conduction studies, quantitative sensory testing, autonomic testing and sympathetic skin response are presented.

(Rev Med Chile 2018; 146: 1079-1084)

Key words: Fabry Disease, Small Fiber Neuropathy, Autonomic Nervous System Diseases.
'Departamento de Neurología y Psiquiatría, Clínica Alemana de Santiago-Universidad del Desarrollo. Santiago, Chile. ${ }^{2}$ Departamento de Ciencias Neurológicas, Facultad de Medicina, Universidad de Chile. Santiago, Chile.

${ }^{3}$ Departamento de Neurociencias, Facultad de Medicina,

Universidad de Chile. Santiago,

Chile.

${ }^{4}$ Instituto Milenio de Neurociencia

Biomedica (BNI), Facultad de Medicina, Universidad de Chile.

Santiago, Chile.

aPhD.

${ }^{\mathrm{b}} \mathrm{MsC}$

Trabajo no recibió financiamiento. Los autores declaran no tener conflictos de interés.

Recibido el 10 de junio de 2018, aceptado el 6 de septiembre de 2018.

Correspondencia a: Dra. Paula Jara M. Avenida Vitacura 5951 Santiago, Chile. pjaram@alemana.cl
L a enfermedad de Fabry (EF) es una enfermedad de depósito lisosomal con herencia ligada al X, con mutaciones que afectan al gen GLA en el locus Xq22.1, causando deficiencia parcial o total de la enzima $\alpha$ galactosidasa A. A nivel mundial la enfermedad tiene una incidencia de 1 en 40.000 a 1 en 117.000 casos $^{1,2}$. Como consecuencia de la deficiencia enzimática se produce acumulación de glicoesfingolípidos en distintos tejidos, incluyendo el sistema nervioso periférico y central. A nivel cutáneo es característica la presencia de angioqueratomas. Las complicaciones tardías de la adultez corresponden a insuficiencia renal, cardiomiopatía y accidente cerebrovascular isquémico ${ }^{1-6}$.

El compromiso del sistema nervioso periférico es la forma de presentación más común y precoz. Típicamente, las fibras aferentes mielínicas de diámetro pequeño (fibras A-delta) y amielínicas (C) están comprometidas. El 70\% de los pacientes presentan crisis de dolor quemante y/o punzante ${ }^{3-6}$. Adicionalmente, pueden presentar disfunción autonómica como intolerancia al calor, hipo/anhidrosis y síntomas gastrointestinales ${ }^{1,4}$.

Presentamos cinco pacientes con EF para analizar el compromiso neurológico periférico tanto clínico como los hallazgos neurofisiológicos (Tablas 1 y 2), incluyendo la evaluación de (i) fibras mielínicas motoras y aferentes de diámetro grueso A-beta (estudio de conducción nerviosa motora y sensitiva) ${ }^{7}$; (ii) fibras aferentes mielinicas de diámetro pequeño A-delta y amielinicas C (análisis de sensibilidad térmica, AST) ${ }^{8-10}$ y (iii) función autonómica (variabilidad del intervalo R-R y respuesta simpática cutánea, RSC) $)^{11-13}$.

\section{Casos clínicos}

\section{Paciente 1}

Hombre de 56 años con antecedentes de EF, hipotiroidismo en tratamiento y miocardiopatía. 

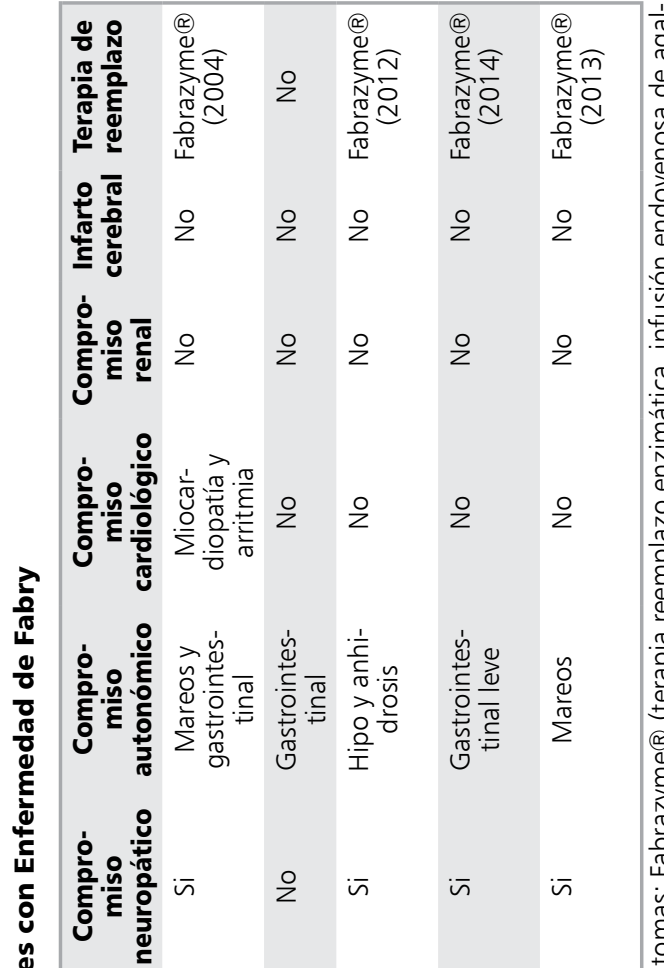

용
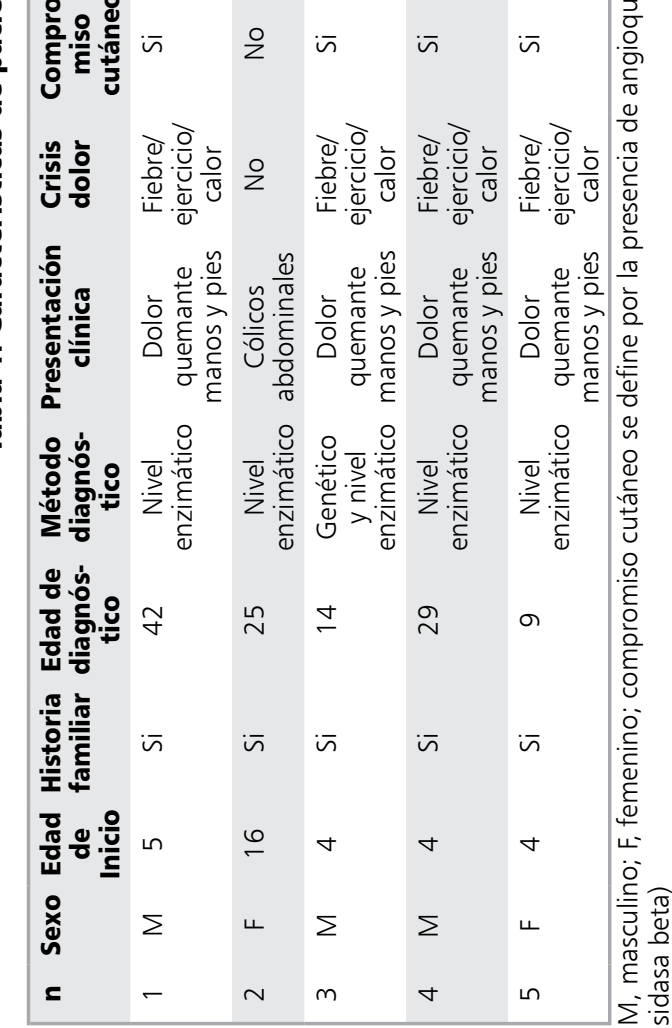

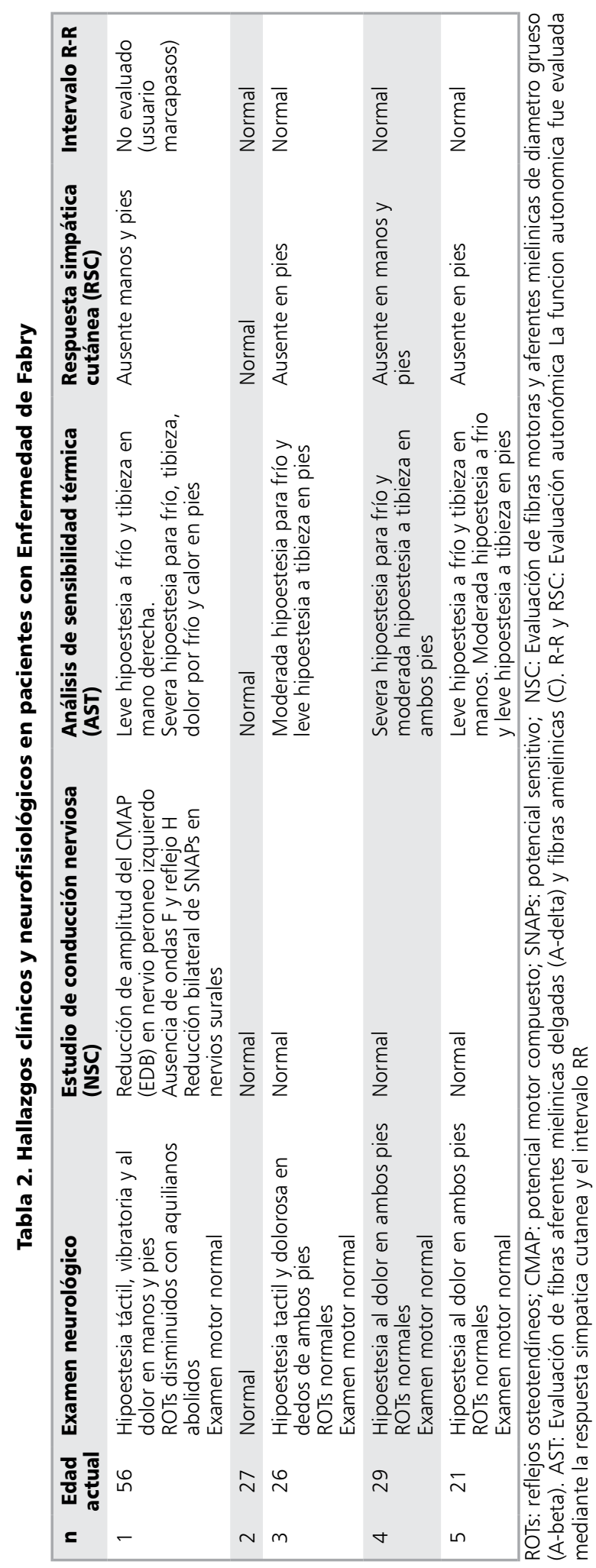


A los 5 años de edad inicia crisis de dolor quemante en manos y pies desencadenadas por ejercicio, exposición al sol y fiebre. Desde los 20 años con disminución progresiva de las crisis. Ocasionalmente sensación de mareo subjetivo y episodios de estitiquez-diarrea. La enfermedad fue diagnosticada a los 42 años como parte de estudio familiar con medición de niveles enzimáticos. Inició terapia de reemplazo enzimático a los 45 años. A los 52 años se diagnostica miocardiopatía hipertrófica y se instala marcapaso. Escasos angioqueratomas. El examen clínico neurológico mostro signos de polineuropatía sensitiva y la evaluación neurofisiológica evidenció una polineuropatía con compromiso sensitivo axonal de fibras mielínicas gruesas y severo compromiso de fibras mielínicas delgadas y amielínicas, además de una radiculopatía L5-S1 izquierda. Ausencia generalizada de RSC. No se realizó evaluación intervalo R-R, debido al uso de marcapasos (Figura 1).

\section{Paciente 2}

Mujer de 27 años migrañosa, EF diagnosticada a la edad de 25 años, en estudió con niveles enzimáticos por antecedente materno de Fabry. Inicia a los 16 años episodios de dolor abdominal intensos tipo cólico no asociado a ingesta de alimentos. Posteriormente evoluciono con episodios de dolor 3 veces al mes, de moderada intensidad y autolimitados. Sin complicaciones de EF, mínimos angioqueratomas. Sin terapia de reemplazo enzimático. Sin síntomas sensitivos. La evaluación clínica y neurofisiológica resulto normal.

\section{Paciente 3}

Hombre de 26 años, desde los 4 años de edad con crisis de dolor quemante en manos y pies asociados a fiebre. Desde la infancia siempre con episodios de indigestión, hipo-anhidrosis, palpitaciones ocasionales. Se diagnóstico EF a los 14 años, con estudio genético y con niveles enzi-

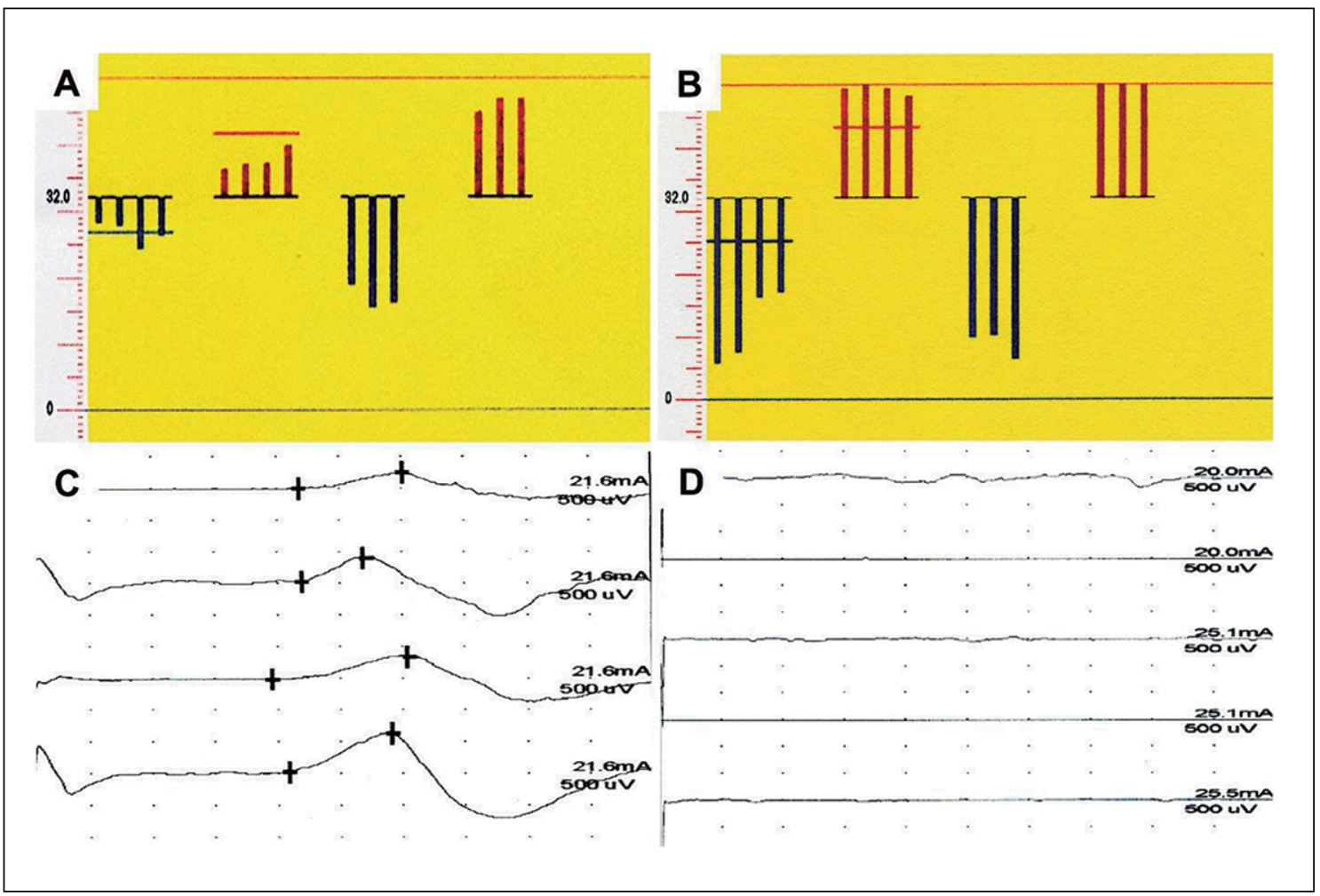

Figura 1. Análisis de sensibilidad térmica (AST) y respuesta simpática cutánea (RSC) en la extremidad inferior derecha en A y C) sujeto sano que presenta umbrales de sensibilidad térmica normales y RSC presente. B y D) paciente con Enfermedad de Fabry. En este caso se detectó una severa neuropatía con hipoestesia para las sensaciones de frío, tibieza, dolor con frío y dolor por calor, asociado a ausencia de RSC. 
máticos. Con el tiempo ha notado mejoría en los síntomas de indigestión. Mantiene basalmente dolor quemante en ambos pies y ocasionalmente presenta crisis de dolor con fiebre. Terapia de reemplazo desde hace 2 años. Tratamiento de dolor neuropático con carbamazepina $400 \mathrm{mg}$ al día. Examen clínico compatible con polineuropatía sensitiva y evaluación neurofisiológica con hallazgos de compromiso de fibras mielínicas delgadas y amielínicas. La RSC ausente en los pies y evaluación intervalo R-R en límites normales.

\section{Paciente 4}

Hombre de 29 años, inicia a la edad de 4 años cuadro caracterizado por crisis dolor quemante en manos y pies, desencadenados por el ejercicio, fiebre, calor o frío. Refiere sensación de pies congelados, para lo cual pone pies en agua tibia o los abriga. Ocasionalmente sensación de pinchazos. Distensión abdominal y episodios de diarrea ocasional. Escasos angioqueratomas. Se realizó el diagnóstico reciente en contexto de estudio familiar, con niveles de enzimáticos. Inicio reciente de terapia de remplazo enzimática. Evaluación clínica y neurofisiológica compatible con compromiso neuropático de fibras mielínicas delgadas y amielínicas. Ausencia generalizada de RSC y evaluación de intervalo R-R en límites normales.

\section{Paciente 5}

Mujer de 21 años, con diagnóstico de EF a los 9 años de edad en contexto de estudio familiar. Síntomas se iniciaron a la edad de 3 a 4 años con dolor quemante en ambos pies, logrando alivio transitorio al colocar sus pies en superficies frías. Crisis de dolor desencadenadas por fiebre y ejercicio. Refiere intolerancia al calor y ocasional sensación de mareos. Escasos angioqueratomas. Terapia de reemplazo iniciada recientemente. Evaluación clínica y neurofisiológica compatible con compromiso neuropático de fibras mielínicas delgadas y amielínicas. Ausencia de RSC en los pies y evaluación intervalo R-R límites normales.

\section{Discusión}

El compromiso neuropático en la EF es muy común, afectando en forma más severa y precoz a los hombres, quienes inician el cuadro a los 9 años de edad en promedio, pudiéndose iniciar precozmente como fue el caso de la mayoria de nuestros pacientes. Típicamente, el síntoma inicial es el dolor quemante en manos y pies, con crisis de dolor que son desencadenadas por calor, estrés, fiebre, fatiga y ejercicio ${ }^{14,15}$. En concordancia a lo descrito en la literatura, nuestros pacientes presentaron hipoestesia táctil, dolorosa y a la tibieza $\mathrm{y}$ al frio, con un patrón de distribución longitud dependiente, con modalidades sensoriales de posición y vibración, reflejos osteotendíneos y examen motor conservados. El estudio de conducción nerviosa fue normal en todos los pacientes (excepción del paciente $\mathrm{N}^{\circ} 1$ ), mientras que el compromiso neuropático de fibra fina se documentó con la alteración de los umbrales de sensibilidad térmica, evidenciándose hipoestesia para las sensaciones de frío y de tibieza en 4 pacientes, con excepción de la paciente No 2 que sólo presentaba algunos síntomas disautonómicos ${ }^{15-17}$ (Figuras 1 y 2 ).

Estudios de AST han mostrado disfunción preferencial para la sensación de frío (A-delta) en el 63 a $100 \%$ de pacientes hombres y en $16-48 \%$ de las mujeres, lo que correspondería a un patrón característico de la enfermedad ${ }^{2,14,16-18}$. A mayor edad disminuyen las crisis de dolor neuropático, lo cual refleja el daño extenso de las fibras sensitivas con perdida de función e hipoalgesia ${ }^{2,3}$. Se ha documentado que en los hombres se produce pérdida completa de función de fibras finas en las extremidades inferiores durante las primeras 2 décadas de vida. En las mujeres en proceso es más tardío y nunca desarrollan pérdida funcional total ${ }^{15}$. Es importante destacar que la EF no produce compromiso de fibras mielínicas gruesas, sin embargo, ciertos pacientes pueden presentarlo tardíamente secundario al compromiso renal o a otra etiología (e.j. paciente $\left.\mathrm{N}^{o} 1\right)^{4,14-16}$. La fisiopatología del compromiso neuropático no es completamente comprendida. Se ha propuesto que la acumulación de glicolípidos se asociaría a reducción de neuronas sensitivas en el ganglio de la raíz dorsal y a reducción selectiva de las fibras A-delta y C detectada en biopsias de nervio sural ${ }^{19,20}$. Adicionalmente se ha documentado disminución de la densidad de fibras intraepidérmicas en patrón longitud dependiente $e^{2,15}$.

Por otra parte, los síntomas de hipo o anhidrosis, trastornos gastrointestinales y alteraciones del ritmo cardiaco que presentan estos pacientes, han sido atribuidos a neuropatía autonómica ${ }^{4,21}$. 


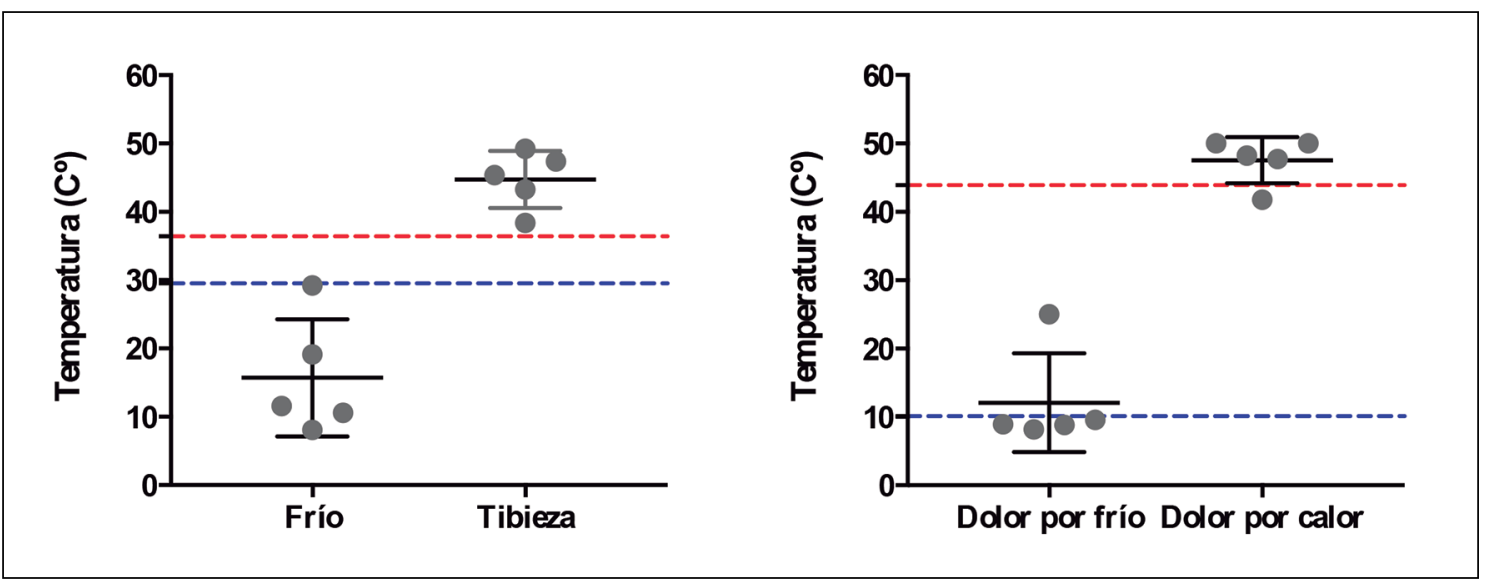

Figura 2. Análisis de sensibilidad térmica (AST) en la extremidad inferior en pacientes con Enfermedad de Fabry. (A) Umbrales de sensibilidad térmica para la sensación de frio y tibieza. (B) Umbrales de sensibilidad térmica para la sensación de dolor por frío y dolor por calor. Cada punto representa el promedio del umbral por cada paciente luego de promediar de 4 determinaciones del umbral de sensibilidad para cada una de las condiciones previamente enumeradas. La líneas punteadas (roja y azul) representan las medias de los valores normales publicados por Verdugo y Ochoa (1992, ref 8).

Sin embargo, si bien tanto en la literatura como en nuestros pacientes, estos síntomas son frecuentemente reportados, no sucede lo mismo con síntomas como intolerancia ortostática y disfunción sexual, los que se encuentran invariablemente en las neuropatías autonómicas. En nuestros pacientes, el estudio de variabilidad del intervalo R-R fue normal. En concordancia con nuestros hallazgos, en estudios previos la evaluación cardiovascular autonómica ha sido normal en la mayoría de los pacientes, excepto en aquellos con miocardiopatía ${ }^{21}$. Con respecto a la RSC, esta se encontró alterada en 4 pacientes, con ausencia de respuesta en manos y pies en los casos No 1 y 4 , y ausencia de respuesta en los pies en los casos No 3 y 5 . Interesantemente, las biopsias de piel no han demostrado reducción en la inervación de las glándulas sudoríparas, sino que acumulación de glicolípidos en estas. El patrón no longitud dependiente de hipo-anhidrosis y el rápido efecto del tratamiento enzimático en la mejoría de estos síntomas sugieren una disfunción local en la glándula ${ }^{4,21}$. Además, como se mencionó previamente, en EF existiría un compromiso relativamente selectivo de las fibras A-delta, por sobre las fibras C. Se plantea entonces, que estos síntomas se debería a un daño terminal de organo blanco por depósito de glicolípidos, más que a un compromiso neuropático de las vías autonómicas ${ }^{15,21}$.
En suma, la EF debe ser considerada como causa de neuropatía de fibra fina de inicio en la infancia y adolescencia. Debido a lo inespecífico de los síntomas iniciales, reporte de síntomas por pacientes de corta edad, variabilidad fenotípica y bajo nivel de sospecha clínica, el diagnóstico suele realizarse luego de muchos años. Por lo tanto, esto retrasaría el inicio de la terapia de remplazo y se favorecería el desarrollo de complicaciones tardias.

\section{Referencias}

1. Zárate YA, Hopkin RJ. Fabry's disease. Lancet 2008; 372: 1427-35.

2. Biegstraaten M, Linthorst GE, Van Schaik IN, Hollak C. Fabry Disease: A Rare Cause of Neuropathic Pain. Curr Pain Headache Rep 2013; 17: 365-71.

3. Burlina AP, Sims KB, Politei JM, Bennet GJ, Baron R, Sommer C, et al. Early diagnosis of peripheral nervous system involvement in Fabry disease and treatment of neuropathic pain: the report of an expert panel. BMC Neurology 2011; 11: 61-72.

4. El-Abassi R, Singhal D, England J. Fabry's disease. Journal of the Neurological Sciences 2014; 344: 5-19.

5. Schiffmann R, Ries M. Fabry Disease: A Disorder of Childhood Onset. Pediatr Neurol 2016; 64: 10-20.

6. Germain D. Fabry Disease. Orphanet Journal of Rare Diseases 2010; 5 (30): 1-49. 
7. Kimura J. Handbook of Clinical Neurophysiology. Peripheral Nerve Diseases Volumen 7 Elsevier 2006.

8. Verdugo R, Ochoa JL. Quantitative Somatosensory Thermotest. A key method for functional evaluation of small calibre afferent channels. Brain 1992; 115: 893913.

9. Yarnitsky D. Quantitative Sensory Testing. Muscle Nerve 1997; 20: 198-204.

10. Backonja NM, Attak N, Baron R, Bouhassira D, Drangholt M, Dyck PJ, et al. Value of quantitative sensory testing in neurological and pain disorders: NeuPSIG consensus. Pain 2013; 154 (9): 1807-19.

11. Vetrugno R, Liguori R, Cortelli P, Montagna P. Sympathetic skin response. Basic mechanism and clinical applications. Clin Auton Res 2003; 13: 256-70.

12. Weimer LH. Autonomic Testing Common Techniques and Clinical Applications. Neurologist 2010; 16: 21522.

13. Hilz M, Dütsch M. Quantitative studies of autonomic function. Muscle Nerve 2006; 33: 6-20.

14. Laaksonen S, Roytta M, Jaaskelainen S, Kantola I, Penttinen M, Falck B. Neuropathic symptoms and findings in women with Fabry disease. Clin Neurophysiol 2008; 119: 1365-72.

15. Biegstraaten M, Hollak C, Bakkers M, Faber C, Aerts J,
Van Schaik I. Small fiber neuropathy in Fabry disease. Mol GenetMetab 2012; 106: 135-41.

16. Dütsch M, Marthol H, Stemper B, Brys M, Haendl T, Hilz MJ. Small Fiber Dysfunction Predominates in Fabry Neuropathy. J Clinical Neurophysiol 2002; 19 (6): 575-86.

17. Luciano C, Russell J, Banerjee T, Quirk J, Scott L, Dambrosia, et al. Physiological characterization of neuropathy in fabry's disease muscle nerve 2002; 26: 622-9.

18. Üçeyler N, Kahn A, Kramer D, Zeller D, Casanova-Molla J, Wanner C, et al. Impaired small fiber conduction in patients with Fabry disease: a neurophysiological case-control study. BMC Neurology 2013; 13: 47-56.

19. Ohnishi A, Dick PJ. Loss of Small Peripheral Sensory Neurons in Fabry Disease. Histologic and Morphometric Evaluation of Cutaneous Nerves, Spinal Ganglia and Posterior Columns. Arch Neurol 1974; 31: 120-7.

20. Gemignani F, Marbini A, Bragaglia M, Govoni E. Pathological Study of the Sural Nerve in Fabrys Disease. Eur Neurol 1982; 23: 173-82.

21. Biegtraaten M, Van Schaik I, Wieling W, Wijburg F, Hollak C. Autonomic neuropathy in Fabry disease: a prospective study using the Autonomic Symptom Profile and cardiovascular autonomic function. BMC Neurology 2010; 10: 38-47. 